\title{
MRI Fuzzy Segmentation of Brain Tissue Using Neighborhood Attraction With Neural-Network Optimization
}

\author{
Shan Shen, William Sandham, Member, IEEE, Malcolm Granat, and Annette Sterr
}

\begin{abstract}
Image segmentation is an indispensable process in the visualization of human tissues, particularly during clinical analysis of magnetic resonance (MR) images. Unfortunately, MR images always contain a significant amount of noise caused by operator performance, equipment, and the environment, which can lead to serious inaccuracies with segmentation. A robust segmentation technique based on an extension to the traditional fuzzy c-means (FCM) clustering algorithm is proposed in this paper. A neighborhood attraction, which is dependent on the relative location and features of neighboring pixels, is shown to improve the segmentation performance dramatically. The degree of attraction is optimized by a neural-network model. Simulated and real brain MR images with different noise levels are segmented to demonstrate the superiority of the proposed technique compared to other FCM-based methods. This segmentation method is a key component of an MR image-based classification system for brain tumors, currently being developed.
\end{abstract}

Index Terms-Improved fuzzy c-means clustering (IFCM), magnetic resonance imaging (MRI), neighborhood attraction, segmentation.

\section{INTRODUCTION}

$\mathbf{I}$ $\mathrm{N}$ THE analysis of medical images for computer-aided diagnosis and therapy, segmentation is often required as a preliminary stage. Medical image segmentation is a complex and challenging task due to the intrinsic nature of the images. The brain has a particularly complicated structure and its precise segmentation is very important for detecting tumors, edema, and necrotic tissues, in order to prescribe appropriate therapy. Magnetic resonance imaging (MRI) is an important diagnostic imaging technique for the early detection of abnormal changes in tissues and organs. It possesses good contrast resolution for different tissues and has advantages over computerized tomography (CT) for brain studies due to its superior contrast properties. Therefore, the majority of research in medical image segmentation concerns MR images.

Many image processing techniques have been proposed for brain MRI segmentation, most notably thresholding, region-growing, and clustering. Since the distribution of tissue

Manuscript received June 29, 2004; revised November 29, 2004 and January 14, 2005.

S. Shen and A. Sterr are with the Department of Psychology, University of Surrey, Guildford GU2 7XH, U.K. (e-mail: shan.shen@surrey.ac.uk; a.sterr@ surrey.ac.uk).

W. Sandham is with Scotsig, Glasgow G12 9PF, U.K. (e-mail: w.sandham @ scotsig.co.uk).

M. Granat is with the School of Health and Social Care, Glasgow Caledonian University, Glasgow G4 0BA, U.K. (e-mail: malcolm.granat@gcal.ac.uk)

Digital Object Identifier 10.1109/TITB.2005.847500 intensities in brain images is very complex, it leads to difficulties of threshold determination. Therefore, thresholding methods are generally restrictive and have to be combined with other methods [1], [2]. Region growing extends thresholding by combining it with connectivity conditions or region homogeneity criteria. Successful methods require precise anatomical information to locate single or multiple seed pixels for each region and together with their associated homogeneity [3]-[5]. Clustering is the most popular method for medical image segmentation, with fuzzy c-means (FCM) clustering and expectation-maximization (EM) algorithms being the typical methods. The applications of the EM algorithm to brain MR image segmentation were reported by Wells et al. [6] and Leemput et al. [7]. A common disadvantage of EM algorithms is that the intensity distribution of brain images is modeled as a normal distribution, which is untrue, especially for noisy images.

The FCM algorithm has also been employed by many researchers. Li et al. [8] presented a knowledge-based classification and tissue labeling approach to initially segment MR brain images using the FCM algorithm, and introduced an expert system to locate a landmark tissue by matching it with a prior model. Hall et al. [9] segmented brain MR images using an artificial neural network (ANN), and compared the performance with FCM. FCM was shown to be superior on normal brains, but worse on abnormal brains with edema, tumor, etc. Pham and Prince [10] extended the traditional FCM algorithm to deal with MR images corrupted by intensity inhomogeneities. Unfortunately, the greatest shortcoming of FCM is its over-sensitivity to noise, which is also a flaw of many other intensity-based segmentation methods. Since medical images always include considerable uncertainty and unknown noise, this generally leads to further degradation with segmentation.

An MR image-based brain tumor classification system is being developed by the authors, and this was the initial motivation to develop a robust segmentation method, since accurate and robust segmentation is a key stage in successful classification. Many extensions of the FCM algorithm have been reported in the literature to overcome the effects of noise, but most of them still have major drawbacks.

In this paper, new extensions to FCM are described which consider two influential factors in segmentation, both of which address issues of neighborhood attraction. One is the feature difference between neighboring pixels in the image; the other is the relative locations of neighboring pixels. Segmentation is therefore decided not only by the pixel intensities themselves, but 
also by the neighboring pixel intensities and locations. Consideration of these neighboring pixels greatly restrains the influence of noise. The parameters referring to the degree of neighborhood attraction are determined using a simple ANN model.

The organization of the paper is as follows. In Section II, the traditional FCM algorithm and related extensions are briefly reviewed. Section III presents our improved fuzzy clustering algorithm, and Section IV compares our method with other published techniques. Section V contains conclusions and addresses future work.

\section{FuZzy C-Means Clustering Algorithm AND RELATED EXTENSIONS}

\section{A. Fuzzy C-Means Clustering (FCM) Algorithm}

Historically, the FCM clustering algorithm introduced by Bezdek is an improvement of earlier clustering methods [11]. It is based on minimizing an objective function, with respect to fuzzy membership $U$, and set of cluster centroids $V$

$$
J_{m}(U, V)=\sum_{j=1}^{N} \sum_{i=1}^{C} \mathbf{u}_{i j}^{m} d^{2}\left(\mathbf{x}_{j}, \mathbf{v}_{i}\right) .
$$

In the above equation, $X=\left\{\mathbf{x}_{1}, \mathbf{x}_{2}, \ldots \mathbf{x}_{j}, \ldots \mathbf{x}_{N}\right\}$ is a $p \times$ $N$ data matrix, where $p$ represents the dimension of each $\mathbf{x}_{j}$ "feature" vector, and $N$ represents the number of feature vectors (pixel numbers in the image). $C$ is the number of clusters. $\mathbf{u}_{i j} \subseteq$ $U(p \times N \times C)$ is the membership function of vector $\mathbf{x}_{j}$ to the $i$ th cluster, which satisfies $\mathbf{u}_{i j} \in[0,1]$ and $\sum_{i=1}^{C} \mathbf{u}_{i j}=1,(j=$ $1,2, \ldots, N)$. The membership function is expressed as follows:

$$
\mathbf{u}_{i j}=\frac{1}{\sum_{k=1}^{C}\left(\frac{d\left(\mathbf{x}_{\mathbf{j}}, \mathbf{v}_{i}\right)}{d\left(\mathbf{x}_{j}, \mathbf{v}_{k}\right)}\right)^{2 /(m-1)}} .
$$

$V=\left\{\mathbf{v}_{1}, \mathbf{v}_{2}, \ldots \mathbf{v}_{i}, \ldots \mathbf{v}_{C}\right\}$, which is a $p \times C$ matrix and denotes the cluster feature center

$$
\mathbf{v}_{i}=\frac{\sum_{j=1}^{N}\left(\mathbf{u}_{i j}\right)^{m} \mathbf{x}_{j}}{\sum_{j=1}^{N}\left(\mathbf{u}_{i j}\right)^{m}} \quad(i=1,2 \ldots C) .
$$

$m \in(1, \infty)$ is a weighting exponent on each fuzzy membership, which controls the degree of fuzziness. $d^{2}\left(\mathbf{x}_{j}, \mathbf{v}_{i}\right)$ is a measurement of similarity between $\mathbf{x}_{j}$ and $\mathbf{v}_{i}$

$$
d^{2}\left(\mathbf{x}_{j}, \mathbf{v}_{i}\right)=\left\|\mathbf{x}_{j}-\mathbf{v}_{i}\right\|^{2} .
$$

$\|\cdot\|$ can be defined as either a straightforward Euclidean distance or its generalization such as the Mahalanobis distance.

The feature vector $X$ in MR images represents the pixel intensities, so $p=1$. The FCM algorithm iteratively optimizes $J_{m}(U, V)$ with the continuous update of $U$ and $V$, until $\left|U^{(l+1)}-U^{(l)}\right| \leq \varepsilon$, where $l$ is the number of iterations.

The drawback of FCM for image segmentation is obvious. First, it is clear from (1) that the objective function of FCM does not take into consideration any spatial dependence among $X$, but deals with images the same as separate points. Secondly, the membership function $U$ in (2) is mostly decided by $d^{2}\left(\mathbf{x}_{j}, \mathbf{v}_{i}\right)$, which measures the similarity between the pixel intensity and the cluster center. Higher membership depends on closer intensity values to the cluster center. It therefore increases the sensitivity of the membership function to noise. If an MR image contains noise or is affected by artefacts, their presence can change the pixel intensities, which will result in an incorrect membership and improper segmentation. These problems must be properly addressed to improve the robustness of the FCM algorithm.

\section{B. FCM-Related Extensions}

The most direct way to compensate for the drawback of FCM is to smooth the image before segmentation. However, standard smoothing filters can lead to a loss of important image details. Various extensions of the FCM algorithm which attempt to accommodate noise have been presented by many researchers. Tilias and Panas post-processed the membership function to smooth the effect of noise [12]. Acton and Mukherjee incorporated multiscale information to enforce spatial constraints [13].

The most popular approach for increasing the robustness of FCM to noise is to modify the objective function directly. Dáve proposed the idea of a noise cluster to deal with noisy clustering data in the approach known as NC [14]. Noise is effectively clustered into a separate cluster which is unique from signal clusters. However, it is not suitable for image segmentation, since noisy pixels should not be separated from other pixels, but assigned to the most appropriate clusters in order to reduce the effect of noise.

Another similar method, developed by Krishnapuram and Keller [15], is called possibilistic c-means (PCM), which interprets clustering as a possibilistic partition. Instead of having one term in the objective function, a second term is included, forcing the membership to be as high as possible without a maximum limit constraint of one. However, it caused clustering being stuck in one or two clusters.

Pham presented a new approach of FCM, named the robust fuzzy c-means algorithm (RFCM) [16]. A modified objective function was proposed for incorporating spatial context into FCM. A parameter controls the tradeoff between the conventional FCM objective function and the smooth membership functions. However, the modification of the objective function results in the complex variation of the membership function.

Other improved versions of FCM by the modification of the objective function were introduced by Ahmed et al. [17] and by Zhang and Chen [18]. The former proposed a modification of the objective function by introducing a term that allows the labeling of a pixel to be influenced by the labels in its immediate neighborhood. The latter (spatially constrained kernelized FCM-SKFCM) used a different penalty term containing spatial neighborhood information in the objective function, and simultaneously the similarity measurement in the FCM, was replaced by a kernel-induced distance. All these methods inevitably introduce computation issues, by modifying most equations along with the modification of the objective function, and have to lose the continuity from FCM, which is well-realized with many types of software, such as MATLAB. 


\section{IMPROVED FUZZY c-MEANS CLUSTERING ALGORITHM}

\section{A. Description of Methodology}

A new algorithm is proposed here in order to circumvent the drawbacks of other methods described above. We have coined it improved fuzzy c-means clustering (IFCM). From the above description, the membership value of FCM decides the segmentation results, and the membership value is determined by the similarity measurement $d^{2}\left(\mathbf{x}_{j}, \mathbf{v}_{i}\right)$ in (4). It may be deduced that this measurement is the key to segmentation success. In FCM, $d^{2}\left(\mathbf{x}_{j}, \mathbf{v}_{i}\right)$ is a measure of the difference between the intensity of a pixel and the cluster center, and has no resistance to noise. In our proposed algorithm, an attraction entitled neighborhood attraction is considered to exist between neighboring pixels. During clustering, each pixel attempts to attract its neighboring pixels toward its own cluster. This neighborhood attraction depends on two factors; the pixel intensities or feature attraction, and the spatial position of the neighbors or distance attraction, which also depends on the neighborhood structure.

IFCM considers the neighborhood attraction in $d^{2}\left(\mathbf{x}_{j}, \mathbf{v}_{i}\right)$ directly

$$
d^{2}\left(\mathbf{x}_{j}, \mathbf{v}_{i}\right)=\left\|\mathbf{x}_{j}-\mathbf{v}_{i}\right\|^{2}\left(1-\lambda H_{i j}-\xi F_{i j}\right)
$$

where $H_{i j}$ represents the feature attraction and $F_{i j}$ represents the distance attraction. The two parameters $\lambda$ and $\xi$ in (5), of magnitude between 0 to 1 , adjust the degree of the two neighborhood attractions

$$
H_{i j}=\frac{\sum_{k=1}^{S} \mathbf{u}_{i k} g_{j k}}{\sum_{k=1}^{S} g_{j k}}
$$

where $g_{j k}$ is the intensity difference between the study pixel $j$ and its neighbor pixel $k$

$$
g_{j k}=\left|\mathbf{x}_{j}-\mathbf{x}_{k}\right| .
$$

$\mathbf{u}_{i k}$ is the membership of neighboring pixel $k$ to the $i$ th cluster, and $S$ is the number of neighboring pixels

$$
F_{i j}=\frac{\sum_{k=1}^{S} \mathbf{u}_{i k}^{2} q_{j k}^{2}}{\sum_{k=1}^{S} q_{j k}^{2}}
$$

where $q_{j k}$ is the relative location between pixel $j$ and its neighborhood pixel $k$.

The neighborhood structure is of the form

$$
K_{j}=\left\{k \in N \mid 0<\left(a_{j}-a_{k}\right)^{2}+\left(b_{j}-b_{k}\right)^{2} \leq Q\right\}
$$

where $\left(a_{j}, b_{j}\right),\left(a_{k}, b_{k}\right)$ denote the coordinates of pixel $j, k . Q$ is a constant, equal to $2^{(L-1)}$, and $L$ is the level of the neighborhood. Fig. 1 shows the neighborhood structure for different levels.

$q_{j k}$ in (7) can be described as follows:

$$
q_{j k}=\left(a_{j}-a_{k}\right)^{2}+\left(b_{j}-b_{k}\right)^{2} .
$$

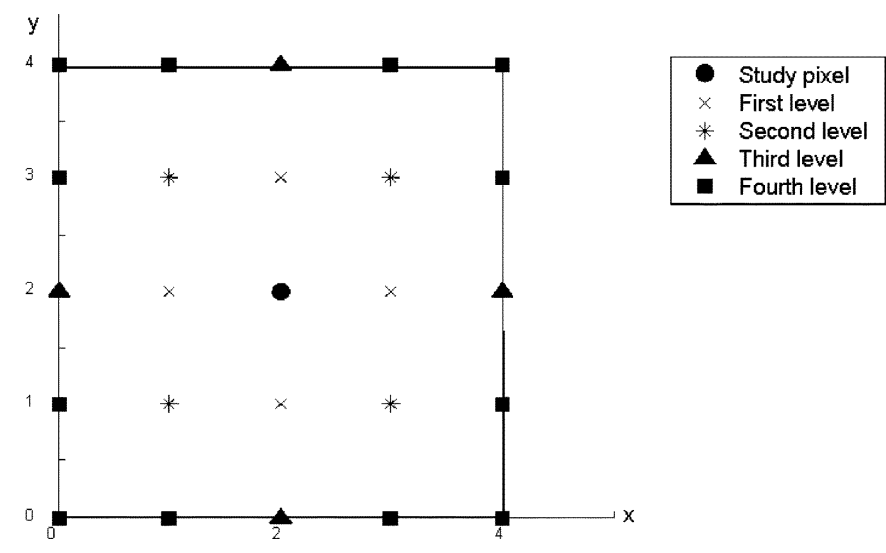

Fig. 1. Neighborhood structure definitions. (A higher level includes pixels labeled as the number of the level and pixels in all lower levels).

The mechanics of the IFCM algorithm are similar to the traditional FCM. Besides modifying $d^{2}\left(\mathbf{x}_{j}, \mathbf{v}_{i}\right)$, the initialization of the membership is not created randomly but inherited from FCM. The individual stages of IFCM are as follows.

1. Determine the number of clusters $C(2 \leq C \leq$

$N)$ and the degree of fuzziness $m$.

2. Execute FCM completely.

3. Utilize the final membership of FCM as the initial membership $\mathbf{u}_{i j}^{(0)}$ of IFCM.

4. At the $l$ th iteration $(l=0,1,2, \ldots)$, calculate the cluster center $\mathbf{v}_{i}^{(l)}(i=1,2 \ldots, C)$ using the membership $\mathbf{u}_{i j}^{(l)}$.

5. Calculate the improved similarity measurement $d^{2}\left(\mathbf{x}_{j}, \mathbf{v}_{i}^{(l)}\right)$.

6. Update $\mathbf{u}_{i j}^{(l)}$ with $d^{2}\left(\mathbf{x}_{j}, \mathbf{v}_{i}^{(l)}\right)$.

7. Compare $\mathbf{u}_{i j}^{(l)}$ and $\mathbf{u}_{i j}^{(l-1)}$, If $\left\|\mathbf{u}_{i j}^{(l)}-\mathbf{u}_{i j}^{(l-1)}\right\|<\varepsilon$, then stop, otherwise, $l=l+1$, go to step 4 and repeat.

\section{B. Parameter Optimization Using Neural Network}

As indicated above, two parameters $\lambda$ and $\xi$ are selected to adjust the degree of feature attraction and distance attraction, respectively, in (5). A simple ANN model is therefore designed to search for the optimal values of these parameters. Fig. 2 shows the architecture of the model, where $\mathbf{w}_{i}(i=0,1,2)$ denotes the interconnection weights of the network

$$
\mathbf{w}=\left[\begin{array}{lll}
w_{0} & w_{1} & w_{2}
\end{array}\right]^{T}=\left[\begin{array}{ll}
1 & \lambda \xi
\end{array}\right]^{T} .
$$

The cost function $E$ of the neural network is described as follows:

$$
E=\frac{1}{N} \sum_{j=1}^{N} \frac{M J_{m}}{\text { DoRate }}
$$

where $j=1 \ldots N$

$$
M J_{m}=\frac{\operatorname{Min}\left(J_{m}\right)}{\operatorname{Max}\left(J_{m}\right)} .
$$




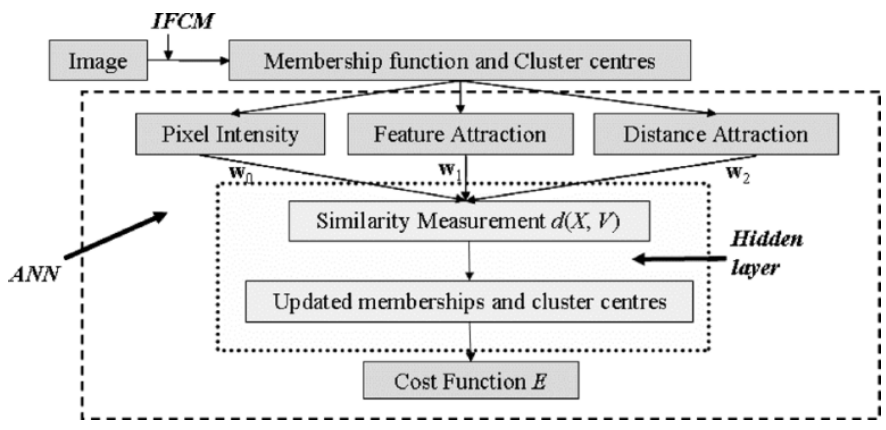

Fig. 2. Architecture of ANN model.

$J_{m}$ is the objective function in (1), which requires to be minimized. It gradually converges during IFCM iteration. Hence, $M J_{m}$ describes the degree of the convergence. A lower $M J_{m}$ represents a better convergence of $J_{m}$

$$
\text { DoRate }=\frac{1}{N} \sum_{j=1}^{N} \operatorname{Max} U_{j} .
$$

DoRate is defined as the abbreviation of "Dominating Rate." $\operatorname{Max} U_{j}$ stands for the highest membership of each pixel to all clusters, thus DoRate represents the average dominating rate in segmentation. Higher DoRate indicates a more pronounced segmentation result.

In the cost function $E$, the numerator $M J_{m}$ is proposed to be minimized and the denominator DoRate is meant to be maximized; hence $E$ must converge to a global minimum in the ANN model. $(1 / N) \sum_{j=1}^{N}$ in $(10)$ denotes an average minimum achieved in the whole image.

The increasing rate of the weights is defined as

$$
\Delta \mathbf{w}=\eta \frac{E}{D}
$$
where $D=\left[\begin{array}{l}D_{1} \\ D_{2}\end{array}\right]=\left[\begin{array}{l}\sum_{i=1}^{C} \sum_{j=1}^{N} H_{i j} \\ \sum_{i=1}^{C} \sum_{j=1}^{N} F_{i j}\end{array}\right], \eta$ is the learning
rate.

The update of $\mathbf{w}$ is then presented as

$$
\mathbf{w}^{(l+1)}=\mathbf{w}^{(l)}+\Delta \mathbf{w}=\mathbf{w}^{(l)}+\eta \frac{\frac{1}{N} \sum_{j=1}^{N} \frac{M J_{m}}{\text { DoRate }}}{D}
$$

where $l$ is the number of iteration times.

The process of parameter optimization includes the following steps.

1. Initialize $\mathbf{w}^{(0)}$, where $\lambda=0$ and $\xi=0$.

2 . Obtain the initial membership function and

the cluster centers.

3. Compute the similarity measurement using

the present $\mathbf{w}^{(l)}$.

4. Update the membership function and the cluster centers and compute the cost function E.

5. Update $\mathbf{w}^{(l)}$ to $\mathbf{w}^{(l+1)}$ using (14).

If $\mathbf{w}_{r}^{(l+1)} \geq 1$, Then $\mathbf{w}_{r}^{(l+1)}=\mathbf{w}_{r}^{(l+1)}-1$, where $r=1,2$.

6. Stop if both $\mathbf{w}_{1,2}^{l+1}$ reached 1 once, otherwise

go to step 3 .

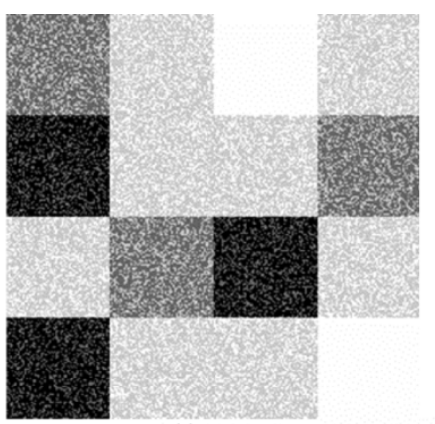

(a)

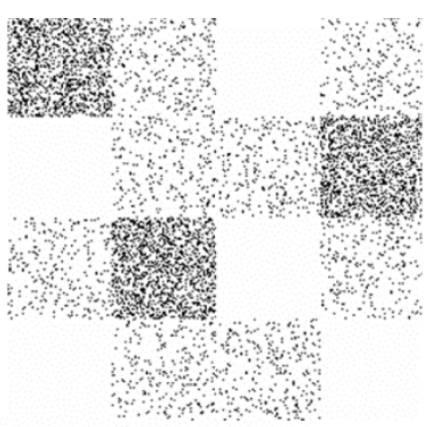

(b)

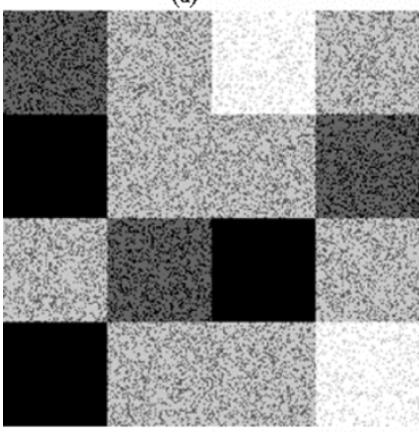

(c)

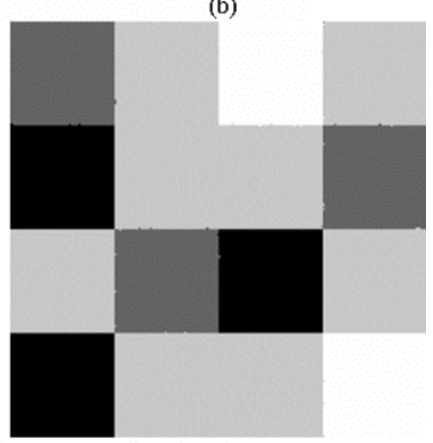

(d)

Fig. 3. Segmentation results on a synthetic square image with $(0,120)$ noise. (a) FCM. (b) PCM. (c) RFCM. (d) IFCM.

The termination criteria are defined as both parameters are searched completely from 0 to 1 . This helps to ensure a global minimum of the cost function is achieved rather than a local minimum.

\section{RESULTS}

In this section, experimental results are described to compare the segmentation performance of IFCM to other FCM extensions. Three types of images were employed: 1) a synthetic square image; 2) simulated brain images downloaded from Brainweb [19] (http://www.bic.mni.mcgill.ca/brainweb); and 3) real MR images from two sources, IBSR [20] (http://www. cma.mgh.harvard.edu/ibsr/) and the Southern General Hospital, Glasgow, U.K.

Unless otherwise stated, a value of 2 was employed for the weighting exponent $m$ in all fuzzy clustering methods in this paper, since this value yielded the best results in most images. The second level of the neighborhood was always used in the IFCM algorithm. Optimized parameters in RFCM were obtained by means of a cross-validation method presented in [16]. This allowed a fair comparison between all methods.

\section{A. Square Image}

A synthetic square image $(256 \times 256$ pixels $)$ consisting of 16 squares is generated. It contains uniformly distributed noise in the interval $(0,120)$. The intensity values of the four classes are $0,100,200$, and 300, respectively. Fig. 3(a)-(d) shows the segmentation results of FCM, PCM, RFCM, and IFCM. $\lambda=$ 0.4675 and $\xi=0.5592$ in IFCM were chosen by the ANN model within 21 iterations, where the learning rate was $\eta=0.2$ (This value of $\eta$ applied to all images in this paper). Fig. 4 shows 


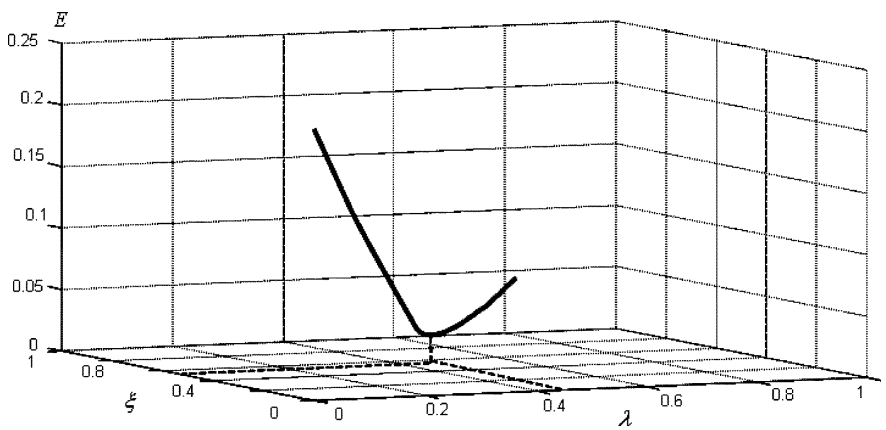

Fig. 4. Parameter selection of IFCM.

TABLE I

SEgmentation Evaluation on Synthetic SQuare IMAGE

\begin{tabular}{c|c|c|c|c}
\hline Methods & FCM & PCM & RFCM & IFCM \\
\hline Parameters & $\mathbf{9 . 5 6}$ & $\mathbf{2 5 . 2}$ & $\mathbf{6 . 4 2}$ & $\mathbf{0 . 0 2 3}$ \\
\hline$U n S(\%)$ & $\mathbf{2 3 . 7 9}$ & $\mathbf{7 5 . 0 0}$ & $\mathbf{1 6 . 2 2}$ & $\mathbf{0 . 0 5 3}$ \\
\hline$O v S(\%)$ & $\mathbf{1 4 . 2 4}$ & $\mathbf{4 3 . 7 5}$ & $\mathbf{9 . 8 8}$ & $\mathbf{0 . 0 2 6}$ \\
\hline $\operatorname{In} C(\%)$ &
\end{tabular}

the trends of the cost function as a function of $\lambda$ and $\xi$ during ANN selection in IFCM. Since the minimum and maximum limits of $\lambda$ and $\xi$ are 0 and 1, respectively, using the ANN model reduces the number of combination possibilities. As shown in Fig. 3(a) and (c), neither FCM nor RFCM can overcome the degradation caused by noise in the segmentation performance. Fig. 3(b) illustrates the drawback of PCM since the segmentation is trapped in two clusters. Only IFCM completely succeeds in segmenting the four classes as shown in Fig. 3(d).

In order to evaluate the segmentation performance quantitatively, some definitions are required. $N_{f p}$ is the number of pixels that do not belong to a cluster and are segmented into the cluster. $N_{f n}$ is the number of pixels that belong to a cluster and are not segmented into the cluster. $N_{p}$ is the number of all pixels that belong to a cluster, and $N_{n}$ is the total number of pixels that do not belong to a cluster. Three evaluation parameters may now be defined as follows.

1) Under segmentation $(U n S): U n S=N_{f p} / N_{n}$, representing the percentage of negative false segmentation.

2) Over segmentation $(O v S): O v S=N_{f n} / N_{p}$, representing the percentage of positive false segmentation.

3) Incorrect segmentation $(\operatorname{InC}): \operatorname{In} C=\left(N_{f p}+N_{f n}\right) / N$, representing the total percentage of false segmentation.

Table I lists the above parameters of the four tested methods. It clearly demonstrates that despite the failure of PCM, RFCM had a better performance than FCM, and that IFCM greatly reduces the incorrect segmentation rate to $0.026 \%$.

\section{B. Simulated MR Images}

Since the ground truth of segmentation for real MR images is not usually available, it is impossible to evaluate the segmentation performance quantitatively, but only visually.

However, Brainweb provides a simulated brain database (SBD) including a set of realistic MRI data volumes produced by an MRI simulator. These data enable us to evaluate the
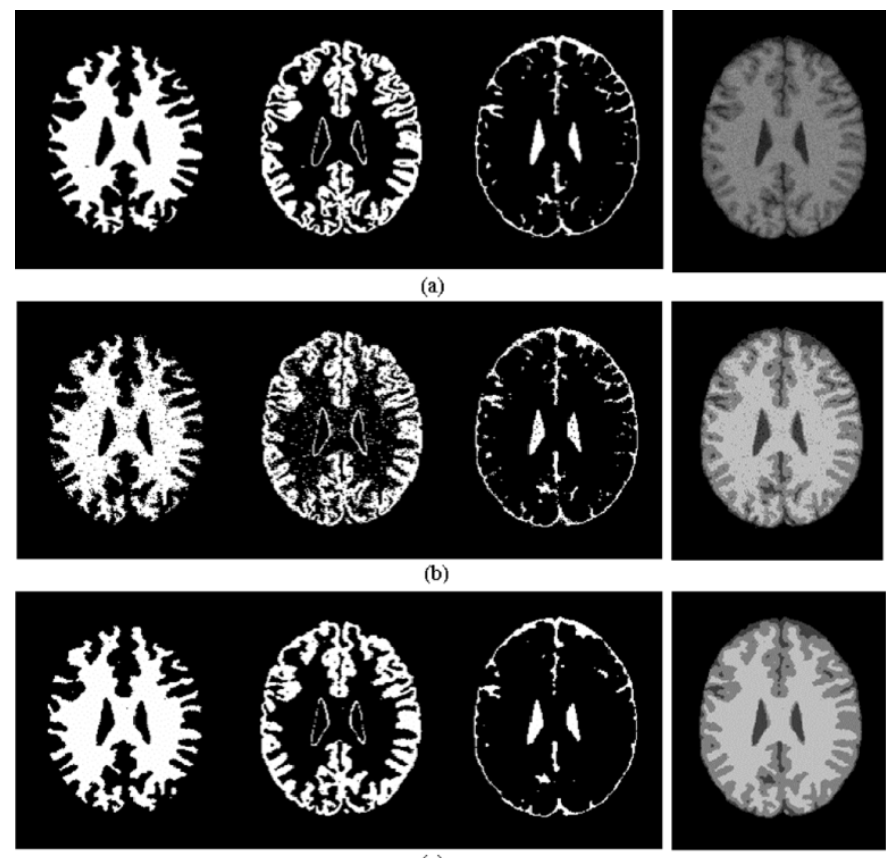

(c)

Fig. 5. Simulated $\mathrm{T}_{1}$-weighted $\mathrm{MR}$ image. (a) Discrete anatomical model (from left to right) white matter, gray matter, CSF, and original image with 7\% noise. (b) Segmentation result of RFCM. (c) Segmentation result of IFCM.

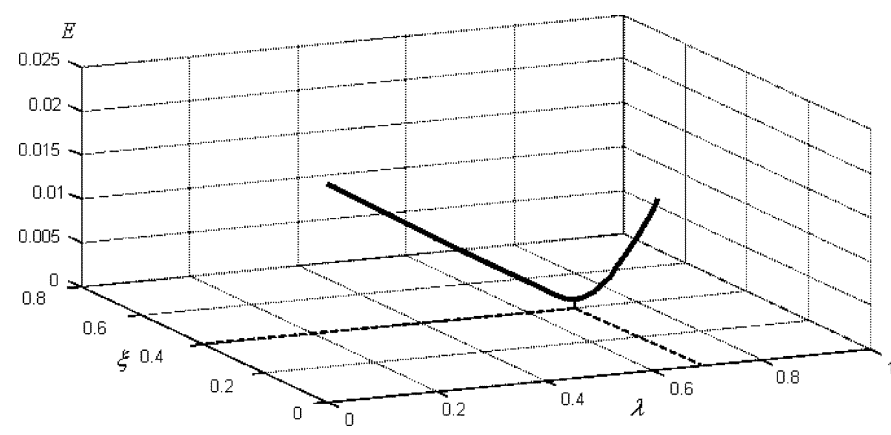

Fig. 6. Parameter optimization in IFCM.

performance of various image analysis methods in a setting where the truth is known [19], [21]-[24].

A simulated $\mathrm{T}_{1}$-weighted MR $(181 \times 217 \times 181)$ image was downloaded from Brainweb. The discrete anatomical model of the simulated image consisting of cerebral spinal fluid (CSF), white matter, and gray matter is shown in Fig. 5(a). 7\% noise was applied to each slice of the simulated image. The noisy 100th brain region only slice is shown in Fig. 5(a). This noisy slice was segmented into four clusters: background, CSF, white matter, and gray matter using FCM, IFCM, PCM, and RFCM; however, the background was neglected from the viewing results. The segmentation results after applying RFCM and IFCM are shown in Fig. 5(b) and (c). Fig. 6 plots the tendency of the cost function in IFCM based on the various values of $\lambda$ and $\xi$. The optimized values in the segmentation were $\lambda=0.7257$ and $\xi=0.4055$, determined over 23 iterations of ANN. A noisy segmentation result was obtained from RFCM, and a clear segmentation result was achieved by IFCM. The performance evaluation parameters of FCM, RFCM, PCM, and IFCM are compiled in Table II. 
TABLE II

SEgmentation Evaluation On Simulated $\mathrm{T}_{1}$-Weighted MR IMAGE

\begin{tabular}{|c|c|c|c|c|c|}
\hline Class & Evaluation Parameters & FCM & PCM & RFCM & IFCM \\
\hline \multirow{3}{*}{ CSF } & $U n S(\%)$ & 0.50 & 0 & 0.47 & 0.20 \\
\hline & $O v S(\%)$ & 7.98 & 100 & 7.98 & 6.82 \\
\hline & $\operatorname{InC}(\%)$ & 0.76 & 34.0 & 0.73 & 0.57 \\
\hline \multirow{3}{*}{$\begin{array}{l}\text { White } \\
\text { matter }\end{array}$} & $\operatorname{UnS}(\%)$ & 1.35 & 0 & 1.11 & 0.95 \\
\hline & $O v S(\%)$ & 11.08 & 100 & 10.92 & 7.31 \\
\hline & $\operatorname{InC}(\%)$ & 2.33 & 10.16 & 2.11 & 1.59 \\
\hline \multirow{3}{*}{$\begin{array}{l}\text { Gray } \\
\text { matter }\end{array}$} & $U n S(\%)$ & 0.75 & 15.86 & 0.76 & 0.54 \\
\hline & $O v S(\%)$ & 7.23 & 0 & 5.72 & 2.65 \\
\hline & $\operatorname{InC}(\%)$ & 1.68 & 13.57 & 1.47 & 0.93 \\
\hline \multirow{3}{*}{ Average } & $\operatorname{UnS}(\%)$ & 0.87 & 5.29 & 0.78 & 0.56 \\
\hline & $O v S(\%)$ & 8.76 & 66.67 & 8.21 & 5.59 \\
\hline & $\operatorname{InC}(\%)$ & 1.59 & 19.24 & 1.44 & 1.03 \\
\hline
\end{tabular}
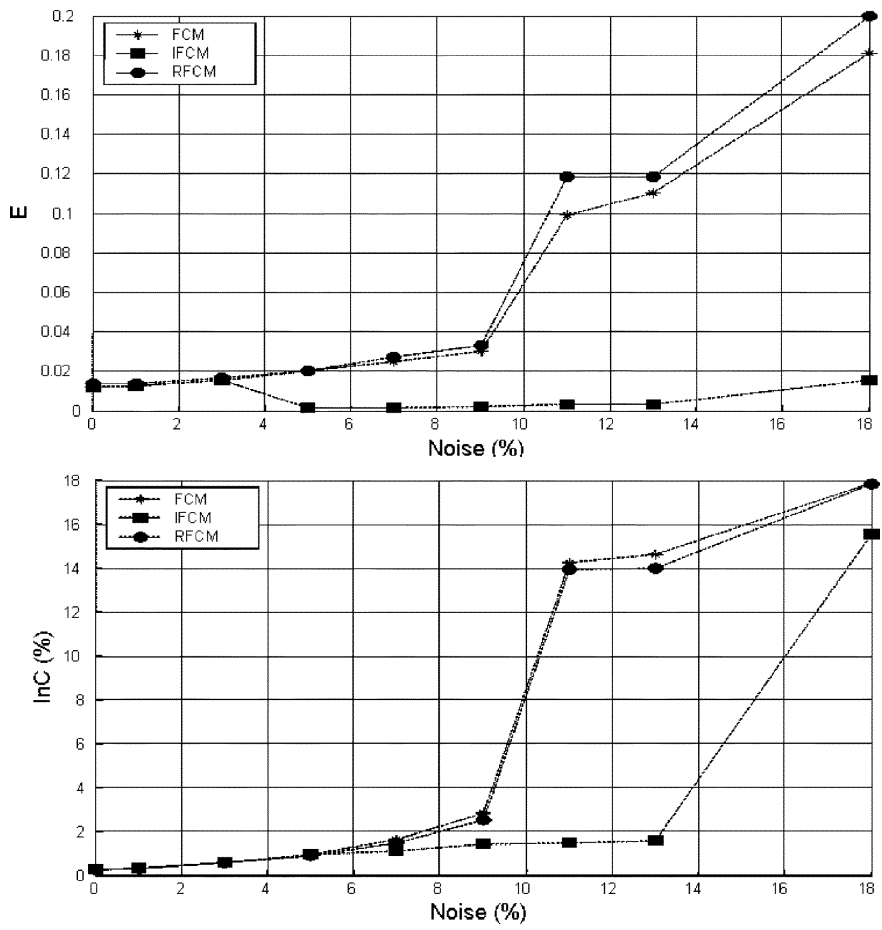

Fig. 7. Variation of (top) cost function $E$, and (bottom) $\operatorname{In} C$ with different noise levels.

To further demonstrate the superior capabilities of IFCM at dealing with noise, different levels $(0 \%-18 \%)$ of noise were sequentially applied to the simulated $\mathrm{T}_{1}$-weighted MR image.

The noisy images were segmented using all methods. Fig. 7 shows the cost functions $E$ and $\operatorname{InC}$ obtained from FCM, RFCM, and IFCM. An increase in the level of noise led to an increase of $E$ and $I n C$ for all methods. Below a $3 \%$ noise level, all three methods had a similar performance described by $E$ and InC. Above 3\% noise, IFCM exhibited the most robustness to noise, and reduced $\operatorname{InC}$ significantly within the noise levels $7 \%-15 \%$. Even with other noise levels, it achieves a much lower $E$, reflecting a more convincing segmentation. The results for RFCM and FCM were close; however, RFCM had a lower $\operatorname{InC}$ and was less convincing in segmentation. This also indicates that the cost function $E$ may be regarded as an approximate measure to $\mathrm{In} C$ for evaluating segmentation
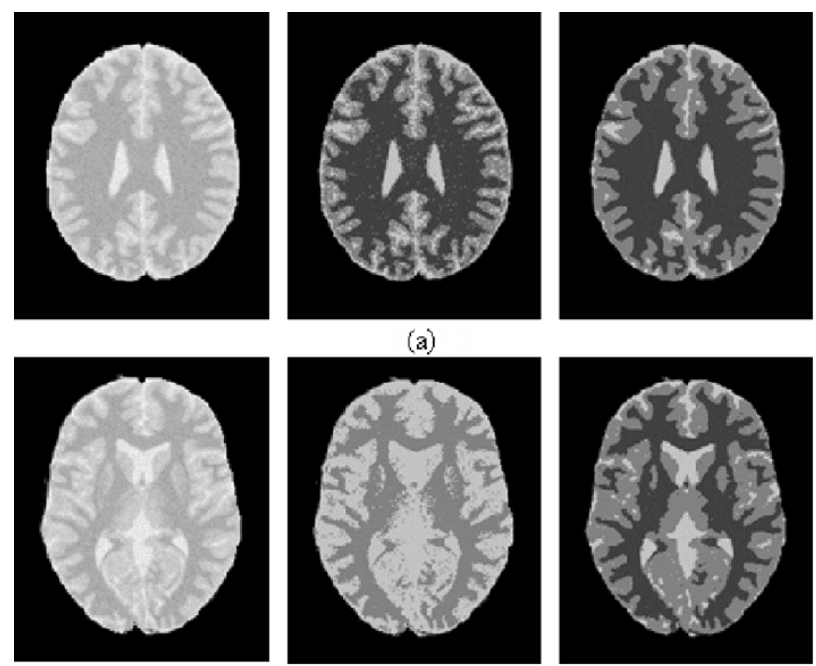

(b)

Fig. 8. Simulated PD-weighted MR image. (a) From left to right: noisy 80th slice, segmentation results of FCM and IFCM. (b) From left to right: noisy 100th slices, segmentation results of FCM and IFCM.

performance, especially when $\operatorname{InC}$ is not obtainable in real images.

A simulated proton density (PD)-weighted image was also used to validate the segmentation advantages of IFCM. $3 \%$ noise was applied to the image (Fig. 8) which was then segmented using both FCM and IFCM (to simplify the comparison, only FCM was compared to IFCM since other methods require an optimization process). Fig. 8(a) and (b) shows the segmentation results. These indicate that whereas FCM is unable to segment the slice correctly, and noise still remains present, IFCM produced the better result with negligible residual noise.

\section{Real MR Images}

The superiority of IFCM was also demonstrated on real MR images. A real MR image was downloaded from IBSR by the Center for Morphometric Analysis at Massachusetts General Hospital. The web provides manually guided expert segmentation results along with brain MRI data to encourage the evaluation and development of segmentation methods. It is abrain only coronal $\mathrm{T}_{1}$-weighted image with a matrix size as $256 \times 256$. The segmentation results of gray and white matter provided were obtained for the spatially normalized scan by trained investigators, using a semi-automated intensity contour mapping algorithm and also using signal intensity histograms [25], [26]. Fig. 9(a) shows the original 25th slice of the image with 3\% Gaussian noise and Fig. 9(b) shows the manual segmentation result provided by the web. For comparison with the manual segmentation result, which included four classes, CSF, gray matter, white matter, and others, the cluster number was set to 4 . The segmentation results of FCM and IFCM are shown in Fig. 9(c) and (d) $(\lambda=0.6038$ and $\xi=0.6097$ within 21 iterations of ANN). Table III lists the evaluation parameters for both methods. IFCM showed a significant improvement over FCM parametrically, and eliminated the effect of noise completely.

A further example of real MR images was chosen from our in-house image database. It is a real $\mathrm{T}_{1}$-weighted image with $11 \%$ additive noise (unsigned 16-bit format). The preprocessing 


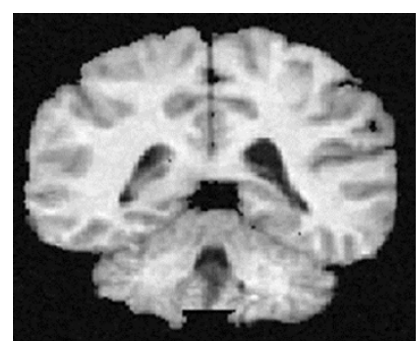

(a)

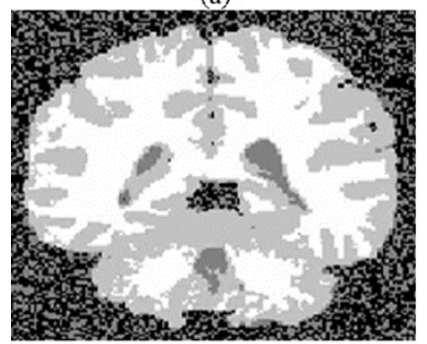

(c)

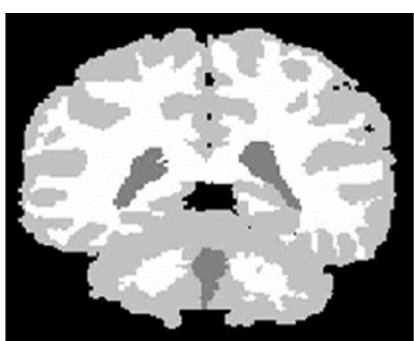

(b)

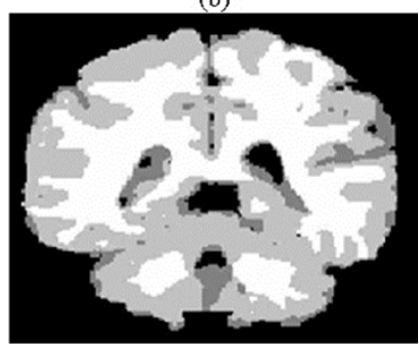

(d)
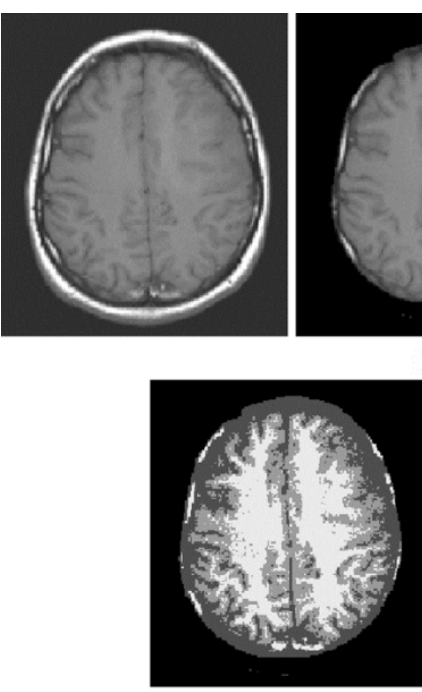

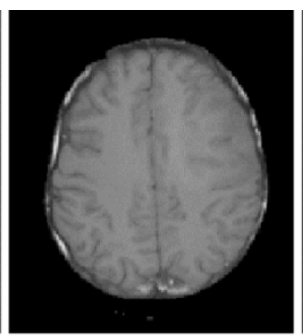

(a)
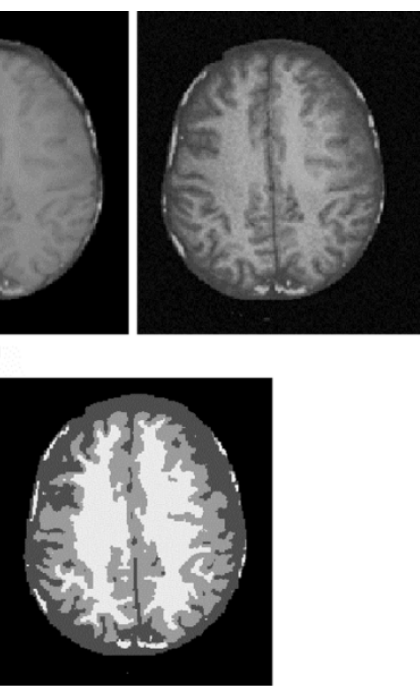

(b)

Fig. 9. $\mathrm{T}_{1}$-weighted image from IBSR. (a) Original image with $3 \%$ noise. (b) Manual segmentation result. (c) Segmentation result of FCM. (d) Segmentation result of IFCM.

TABLE III

SEGMENTATION EVALUATION ON $\mathrm{T}_{1}$-WEIGHTED MR IMAGE

\begin{tabular}{|c|c|c|c|}
\hline Class & Evaluation Parameters & FCM & IFCM \\
\hline \multirow{3}{*}{ CSF } & $U n S(\%)$ & 2.7 & 1.58 \\
\hline & $O v S(\%)$ & 48.22 & 39.64 \\
\hline & $\operatorname{InC}(\%)$ & 3.13 & 1.94 \\
\hline \multirow{3}{*}{$\begin{array}{l}\text { White } \\
\text { matter }\end{array}$} & $\operatorname{UnS}(\%)$ & 0.51 & 0.49 \\
\hline & $O v S(\%)$ & 12.11 & 13.26 \\
\hline & $\operatorname{InC}(\%)$ & 1.40 & 1.47 \\
\hline \multirow{3}{*}{$\begin{array}{l}\text { Gray } \\
\text { matter }\end{array}$} & $\operatorname{UnS}(\%)$ & 1.00 & 1.14 \\
\hline & $O v S(\%)$ & 31.61 & 20.57 \\
\hline & $\operatorname{InC}(\%)$ & 4.05 & 3.07 \\
\hline \multirow{3}{*}{ Average } & $\operatorname{UnS}(\%)$ & 1.40 & 1.07 \\
\hline & $O v S(\%)$ & 30.65 & 24.49 \\
\hline & $\operatorname{In} C(\%)$ & 2.86 & 2.16 \\
\hline
\end{tabular}

steps including standardization, nonbrain region removal, and contrast enhancement were applied to this image before segmentation. Nonstandard MRI intensity scales for same tissue types in different images leads to impossible intensity-based classification. The traditional standardization method scales each MR image into the same maximum and minimum intensity values [27]. However, this is inappropriate to the classification approaches where the intensities may indicate the pathologic information. We proposed a method which standardizes MR images into a prespecified standard deviation and mean; therefore each standardized image had a similar histogram but also retained its original intensity features [28]. Nonbrain regions were also removed using morphological operations and the intensity contrast between white matter and gray matter was enhanced using histogram equalization [29].

The preprocessed image was then segmented using the FCM and IFCM algorithms. The parameters $\lambda$ and $\xi$ in IFCM were obtained as 0.5303 and 0.5707 , using the ANN model within 19 iterations. The cluster number was 4 . The segmentation results along with the preprocessing demonstration are shown in Fig. 10. Clearly, IFCM shows a superior performance than FCM. Both algorithms were also applied to the image with

Fig. 10. $\mathrm{T}_{1}$-weighted MR image with $11 \%$ noise. (a) From left to right: original standardized image, brain only, and enhanced image. (b) From left to right: segmentation results of FCM and IFCM.

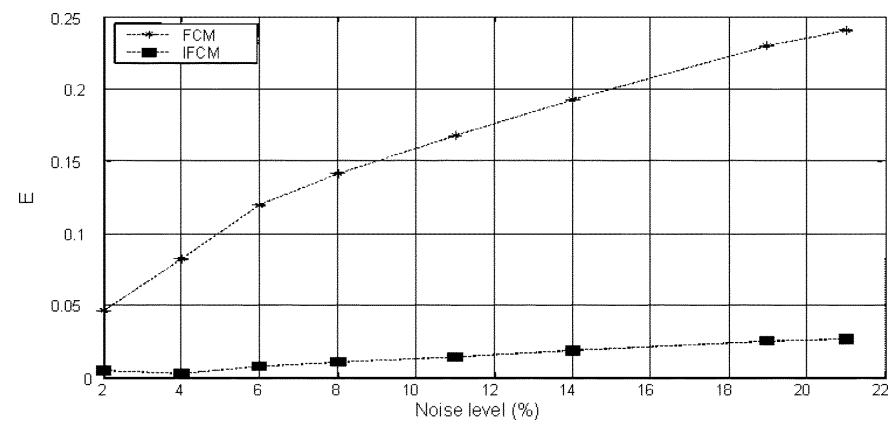

Fig. 11. Variation of $E$ with noise levels.

different noise levels. As no ground truth was available, the cost function $E$ was employed as a performance evaluation parameter. Fig. 11 shows how the value of $E$ increases with the noise level. A lower value of $E$ reflects the superior performance of IFCM compared to FCM.

Finally, the segmentation performance of IFCM was compared with the most recent published algorithm, SKFCM [18], and also other methods whose segmentation results were reported at IBSR [20]. A comparison score (also called overlap metric) used in both is introduced by the following equation:

$$
S_{i}=\frac{A_{i} \cap A_{\mathrm{ref} i}}{A_{i} \cup A_{\mathrm{ref} i}}
$$

where $A_{i}$ represents the pixels belong to the $i$ th class and $A_{\text {ref }} i$ represent the $i$ th reference class as the ground truth. In [18], a simulated $\mathrm{T}_{1}$-weighted image with $3 \%$ noise was segmented by four methods. The highest score obtained among all clusters was 0.88 . The IFCM algorithm was tested on the same image and scored as 0.88 (CSF), 0.85 (gray matter), and 0.92 (white matter).

IBSR reported average scores of various methods such as FCM, maximum aposteriori probability (MAP), biased MAP, adaptive MAP, maximum likelihood, and tree-structure k-means, applied to a dataset including 20 normal brain $\mathrm{T}_{1}$-weighted MR images [20]. The highest score for gray 
matter is 0.56 (adaptive MAP) and for white matter is 0.57 (tree-structure k-means). IFCM was applied to the same dataset, and the average scores obtained were 0.53 (gray matter) and 0.64 (white matter).

As tested on simulated MR images, IFCM reduced InC greatly within the noise levels 7\%-15\%. Therefore, a more significant improvement over other methods can be expected from IFCM at higher noise levels.

\section{CONCLUSION}

Medical images generally contain unknown noise and considerable uncertainty, and therefore clinically acceptable segmentation performance is difficult to achieve. Traditional FCM is a popular segmentation method for medical images. However, it is an intensity-based clustering algorithm which is not robust to noisy images. Although many extended algorithms based on FCM have been developed to overcome this shortcoming, none of them are flawless. Usually, one pixel is too small to represent part of an image. Assuming a pixel has a completely different intensity from its surrounding pixels, it is reasonable to conclude that this pixel must be affected by noise. Its real intensity should be identical to its neighboring pixels. Therefore, it is assumed that an attraction exists between neighboring pixels, which we coin "neighborhood attraction" in this paper. With our proposed IFCM algorithm, during clustering, each pixel attempts to attract its neighboring pixels toward its own cluster.

If a pixel has a very similar intensity to one of its neighbors, the attraction between them should be stronger than the attraction between the pixel and another neighbor with rather different intensities. A spatially closer neighboring pixel should also have a stronger attraction than a neighbor which is spatially distant. The components of the neighborhood can also influence the attraction. Including neighborhood attraction, segmentation using IFCM is not only decided by the pixel itself but also by its neighboring pixels.

In MR images, the presence of noise or artefacts can change the intensities of some pixels; these may be segmented more appropriately with the help of their neighboring pixels. Instead of modifying the objective function, the measurement of similarity was extended by considering neighborhood attraction. This included feature attraction and distance attraction, which account for feature differences and relative spatial locations between neighboring pixels in the image. Compared to RFCM and other FCM-based methods, which modified the objective function and resulted in complex membership functions, IFCM conserved all formulas in FCM except for $d^{2}\left(\mathbf{x}_{j}, \mathbf{v}_{i}\right)$. Therefore, IFCM offers better continuity compared to the traditional FCM algorithm, and simpler computation. Additionally, the fundamental basis of IFCM is more straightforward and easier to understand.

Two parameters $\lambda$ and $\xi$ control the degree of two factors in the neighborhood attraction. A higher $\lambda$ leads to a stronger feature attraction and a higher $\xi$ leads to a stronger distance attraction. Optimized values of these parameters enable the best segmentation results to be achieved. However, inappropriate values can be detrimental. An ANN model was developed to search for optimum values of $\lambda$ and $\xi$, using a cost function decided by the objective function of segmentation and the dominating rate. The model generally finds the optimized parameter within
30 iterations. It reduces the test validation times to $0.2 \%-0.3 \%$ of initial estimates. Images with the same MR modality can use the same values of $\lambda$ and $\xi$, especially after standardization. The cost function of ANN can either validate the optimization of the parameters or even evaluate the segmentation performance.

FCM, IFCM, and other FCM-based algorithms were tested on a synthetic square image, simulated and real MR images, with different noise levels. Three evaluation parameters were defined and measured the segmentation performance quantitatively on simulated images and real images from IBSR due to the availability of ground truth. The cost function in the ANN model was used to validate the results on real images where no ground truth was available. Both quantitative performance and subjective visual comparisons clearly demonstrated the superiority of the proposed IFCM algorithm. A comparison score was introduced to compare the results of IFCM to the newly published FCM based method and also other methods reported by IBSR. IFCM scored higher than most other methods.

Future work will focus on developing the automatic image based classification system for brain tumor using data mining. Preprocessing techniques will be enhanced that brain model fitting may be considered to do the nonbrain region removal. More comprehensive comparison of IFCM and the generalization of the ANN model will be addressed.

\section{ACKNOWLEDGMENT}

The authors would like to thank Dr. M. F. Dempsey and Dr. J. Patterson of the Southern General Hospital, Glasgow, U.K., for providing MR images and clinical advice. The authors would also like to express their sincere appreciation to the reviewers for their careful and thorough critical appraisement of an earlier draft of the paper.

\section{REFERENCES}

[1] H. Suzuki and J. Toriwaki, "Automatic segmentation of head MRI images by knowledge guided thresholding," Comput. Med. Imag. Graph., vol. 15 , no. 4, pp. 233-240, 1991.

[2] L. Lemieux, G. Hagemann, K. Krakow, and F. G. Woermann, "Fast, accurate, and reproducible automatic segmentation of the brain in T1-weighted volume MRI data," Magn. Reson. Med., vol. 42, pp. 127-135, 1999.

[3] A. R. Robb, Biomedical Imaging, Visualization, and Analysis. New York: Wiley, 2000.

[4] R. Pohle and K. D. Toennies, "Segmentation of medical images using adaptive region growing," Proc. SPIE-Med. Imag., vol. 4322, pp. 1337-1346, 2001.

[5] T. Y. Law and P. A. Heng, "Automated extraction of bronchus from 3D CT images of lung based on genetic algorithm and 3D region growing," Proc. SPIE-Med. Imag., vol. 3979, pp. 906-916, 2000.

[6] W. M. Wells III, W. E. L. Grimson, R. Kikinis, and F. A. Jolesz, "Adaptive segmentation of MRI data," IEEE Trans. Med. Imag., vol. 15, no. 4, pp. 429-442, Aug. 1996.

[7] K. V. Leemput, F. Maes, D. Vandermeulen, and P. Suetens, "Automated model-based tissue classification of MR images of the brain," IEEE Trans. Med. Imag., vol. 18, no. 10, pp. 897-908, Oct. 1999.

[8] C. L. Li, D. B. Goldgof, and L. O. Hall, "Knowledge-based classification and tissue labeling of MR images of human brain," IEEE Trans. Med. Imag., vol. 12, no. 4, pp. 740-750, Apr. 1993.

[9] L. O. Hall, A. M. Bensaid, L. P. Clarke, R. P. Velthuizen, M. S. Silbiger, and J. C. Bezdek, "A comparison of neural network and fuzzy clustering techniques in segmenting magnetic resonance images of the brain,” IEEE Trans. Neural Netw., vol. 3, no. 5, pp. 672-682, Sep. 1992.

[10] D. L. Pham and J. L. Prince, "Adaptive fuzzy segmentation of magnetic resonance images," IEEE Trans. Med. Imag., vol. 18, no. 9, pp. 737-752, Sep. 1999. 
[11] J. C. Bezek, Pattern Recognition with Fuzzy Object Function Algorithms. New York: Plenum, 1981.

[12] Y. A. Tolias and S. M. Panas, "On applying spatial constraints in fuzzy image clustering using a fuzzy rule-based system," IEEE Signal. Process. Lett., vol. 5, no. 10, pp. 245-247, Oct. 1998.

[13] S. T. Acton and D. P. Mukherjee, "Scale space classification using area morphology," IEEE Trans. Image Process., vol. 9, no. 4, pp. 623-635, Apr. 2000.

[14] R. N. R. N. Dave, "Characterization and detection of noise in clustering," Pattern Recognit. Lett., vol. 12, pp. 657-664, 1991.

[15] R. R. Krishnapuram and J. M. Keller, "A possibilistic approach to clustering," IEEE Trans. Fuzzy Syst., vol. 1, no. 2, pp. 98-110, May 1993.

[16] D. L. Pham, "Spatial models for fuzzy clustering," Comput. Vis. Imag. Understand., vol. 84, pp. 285-297, 2001.

[17] M. N. Ahmed, S. M. Yamany, N. Mohamed, A. A. Farag, and T. Moriarty, "A modified fuzzy c-means algorithm for bias field estimation and segmentation of MRI data," IEEE Trans. Med. Imag., vol. 21, no. 3, pp. 193-199, Mar. 2002.

[18] D. Q. Zhang and S. C. Chen, "A novel kernelized fuzzy c-means algorithm with application in medical image segmentation," Artif. Intell. Med., vol. 32, pp. 37-52, 2004.

[19] BrainWeb [Online]. Available: www.bic.mni.mcgill.ca/brainweb/

[20] IBSR [Online]. Available: http://www.cma.mgh.harvard.edu/ibsr/

[21] C. A. Cocosco, V. Kollokian, R. K. -S. Kwan, and A. C. Evans, "BrainWeb: Online interface to a 3D MRI simulated brain database," NeuroImage, pt. 2/4, vol. 5, no. 4, p. S425, 1997

[22] R. K. S. Kwan, A. C. Evans, and G. B. Pike, "An extensible MRI simulator for post-processing evaluation," in Visualization in Biomedical Computing (VBC'96). New York: Springer-Verlag, 1996, vol. 1131, Lecture Notes in Computer Science, pp. 135-140.

[23] — , "MRI simulation-based evaluation of image-processing and classification methods," IEEE Trans. Med. Imag., vol. 18, no. 11, pp. 1085-1097, Nov. 1999.

[24] D. L. Collins, A. P. Zijdenbos, V. Kollokian, J. G. Sled, N. J. Kabani, C. J. Holmes, and A. C. Evans, "Design and construction of a realistic digital brain phantom," IEEE Trans. Med. Imag., vol. 17, no. 3, pp. 463-468, Jun. 1998

[25] D. N. Kennedy, P. A. Filipek, and V. S. Caviness, "Anatomic segmentation and volumetric calculations in nuclear magnetic resonance imaging," IEEE Trans Med. Imag., vol. 8, no. 1, pp. 1-7, Mar. 1989.

[26] P. A. Filipek, C. Richelme, D. N. Kennedy, and V. S. Caviness, "The young adult human brain: An MRI-based morphometric analysis," Cereb. Cortex, vol. 4, no. 4, pp. 344-360, 1994.

[27] L. G. Nyúl and J. K. Udupa, "On standardizing the MR image intensity scales," Magn. Reson. Imag., vol. 42, pp. 1072-1081, 1999.

[28] S. Shen, W. A. Sandham, and M. H. Granat, "Preprocessing and segmentation of magnetic resonance imaging," in Proc. 4th IEEE Annu. Int. Conf. Inform. Technol. Appl. Biomed., ITAB'03, U.K., 2003, pp. $149-152$.

[29] S. Shen, W. A. Sandham, M. H. Grant, J. Patterson, and M. F. Dempsey, "Fuzzy clustering based applications to medical image processing," in Proc. IEEE EMBS 25th Annu. Int. Conf., 2003, pp. 747-750.

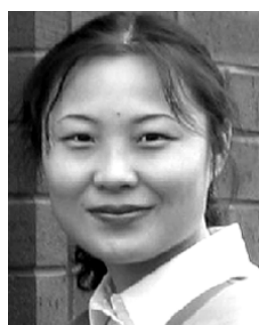

Shan Shen received the B.S. and M.S. degrees in communication engineering and biomedical engineering from the University of Chongqing, China, in 1997 and 2000, and the Ph.D. degree in electronic engineering and bioengineering from the University of Strathclyde, U.K., in 2004.

In 2003, she joined the Department of Psychology, University of Surrey, Guildford, U.K., where she is currently a Research Fellow. Her research interests include structural and functional MR image analysis, medical imaging, genetic programming, and neural

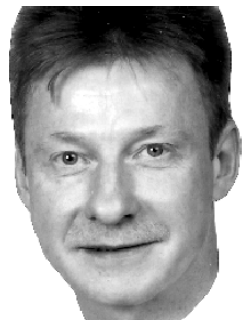

William Sandham (M'86) received the B.Sc.(Hons.) degree from the University of Glasgow, Glasgow, U.K., in 1974 and the Ph.D. degree from the University of Birmingham, Birmingham, U.K., in 1981.

He was a Medical Physicist in the Department of Clinical Physics and Bioengineering, Glasgow, from 1974 to 1976, and was a Geophysicist with the British National Oil Corporation and Britoil from 1980 to 1986. From 1986 to 2003, he was a Lecturer/Senior Lecturer/Reader at the University of Strathclyde, Glasgow. He is currently Managing Director of Scotsig, Glasgow, a signal processing consultancy/training/research company. His current biosignal research interests include MRI segmentation, ECG data analysis and compression, blood glucose prediction for diabetics, sleep apneoa detection, prosthetic limb fitting, and medical ultrasound. He has published over 130 technical papers and five books.

Dr. Sandham has served on the editorial boards of the IEEE TRANSACTIONS ON Circuits AND SyStems PART II-ANalog AND Digital Signal PROCESSING (TCAS-II), and the EURASIP Journal on Applied Signal Processing. He has been an invited lecturer at a number of research institutions across the U.K., Europe, and South America, and has acted as a consultant for British Gas, BP, Shell EXPRO, Appleton Laboratory, and C-CORE (Newfoundland), among others. He has served on the conference organizing committees of ICASSP (Glasgow, 1989), TA-91 (Glasgow, 1991), and EUSIPCO (Edinburgh, 1994).

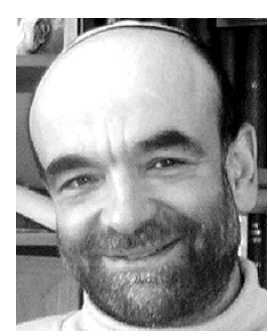

Malcolm Granat received the B.Sc. degree in medical biochemistry from the University of Birmingham, Birmingham, U.K., in 1980 and the Ph.D. degree in bioengineering from the University of Strathclyde, Glasgow, U.K., in 1990. The subject of his $\mathrm{Ph} . \mathrm{D}$. was the application of functional electrical stimulation to restore walking in incomplete spinal cord injured people.

He was a secondary school teacher for six years. $\mathrm{He}$ is currently Chair of Research in the School of Health and Social Care, Glasgow Caledonian University, Glasgow, U.K. His major areas of interest are the development of functional electrical stimulation (FES) based systems for the restoration of movement in neurological conditions, the clinical evaluation of novel therapies, and the development of ambulatory monitoring systems. He has published extensively in these areas.

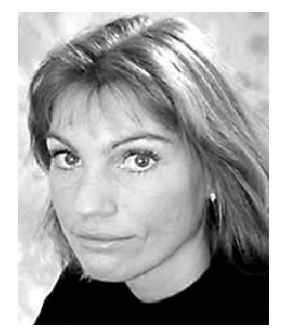

Annette Sterr received degrees in psychology and biology in 1995 and the Ph.D. degree in 1998 from the University of Konstanz, Germany.

She was a Research Fellow at the University of California, San Diego, from 1995 to 1996. From 2000 to 2003, she was a Lecturer/Reader at the University of Liverpool, U.K., where she established the Luria Laboratory for Clinical Neuroscience. She is currently the Chair in Cognitive Neuroscience \& Neuropsychology, University of Surrey, Guildford, U.K. She has published in high-ranking journals such as Nature, Journal of Neuroscience and Neurology. Her research interests include constraint-induced movement therapy, motor rehabilitation, neuropsychology of acquired brain injury, cognition, and functional brain organization.

Dr. Sterr received Young Scientist awards in Japan and Germany in 1997 and 1998. She was chosen by the MRC as one of their Career Development Fellows in 2002 . 